\title{
SCIENCE WITH A WIDE-FIELD UV TRANSIENT EXPLORER
}

\author{
I. Sagiv ${ }^{1}$, A. Gal-Yam ${ }^{1}$, E. O. Ofek ${ }^{1}$, E. Waxman ${ }^{1}$, O. Aharonson ${ }^{2}$, S. R. Kulkarni ${ }^{3}$, E. NaKaR ${ }^{4}$, D. MaOz ${ }^{4}$, \\ B. Trakhtenbrot ${ }^{1}$, E. S. Phinney ${ }^{3}$, J. Topaz ${ }^{1}$, C. Beichman ${ }^{5}$, J. Murthy ${ }^{6}$, and S. P. Worden ${ }^{7}$ \\ ${ }^{1}$ Benoziyo Center for Astrophysics, Weizmann Institute of Science, 76100 Rehovot, Israel \\ ${ }^{2}$ Helen Kimmel Center for Planetary Science, Weizmann Institute of Science, 76100 Rehovot, Israel \\ ${ }^{3}$ Division of Physics, Mathematics, and Astronomy, California Institute of Technology, Pasadena, CA 91125, USA \\ ${ }^{4}$ School of Physics and Astronomy, Tel Aviv University, 93387 Tel Aviv, Israel \\ ${ }^{5}$ Division of Geophysics and Planetary Science, California Institute of Technology, Pasadena, CA 91105, USA \\ ${ }^{6}$ Indian Institute of Astrophysics, Koramangala, Bangalore 560034, India \\ ${ }^{7}$ NASA Ames Research Center, Moffett Field, CA 94035, USA \\ Received 2013 May 20; accepted 2014 January 13; published 2014 March 11
}

\begin{abstract}
The time-variable electromagnetic sky has been well-explored at a wide range of wavelengths. In contrast, the ultra-violet (UV) variable sky is relatively poorly explored, even though it offers exciting scientific prospects. Here, we review the potential scientific impact of a wide-field UV survey on the study of explosive and other transient events, as well as known classes of variable objects, such as active galactic nuclei and variable stars. We quantify our predictions using a fiducial set of observational parameters which are similar to those envisaged for the proposed ULTRASAT mission. We show that such a mission would be able to revolutionize our knowledge about massive star explosions by measuring the early UV emission from hundreds of events, revealing key physical parameters of the exploding progenitor stars. Such a mission would also detect the UV emission from many tens of tidal-disruption events of stars by supermassive black holes at galactic nuclei and enable a measurement of the rate of such events. The overlap of such a wide-field UV mission with existing and planned gravitational-wave and high-energy neutrino telescopes makes it especially timely.
\end{abstract}

Key words: binaries: eclipsing - methods: observational - supernovae: general - surveys - ultraviolet: general

Online-only material: color figures

\section{INTRODUCTION}

The coming decade is expected to be a golden age for timedomain astronomy, which has been identified as an area of unusual discovery potential by the 2010 Decadal Survey.

There are three reasons for this developing focus. In most electromagnetic (EM) bands the static sky has been imaged to interesting depths: FIRST (Becker et al. 1995) and NVSS (Condon et al. 1998) in radio, 2MASS (Skrutskie et al. 2006), UKIDSS (Lawrence et al. 2007) and WISE (Wright et al. 2010) in IR, Sloan Digital Sky Survey (SDSS; York et al. 2000) in optical, GALEX AIS in UV (Martin et al. 2005), and ROSAT (Voges et al. 1999) in X-rays.

Next, technology is now enabling efficient monitoring of large swaths of sky. Advances include arrays of sensitive detectors in the IR, visible and UV, as well as increase in computing power, data storage capacity, and improved communications. Following advances in astronomical software designed for handling large-area static surveys, new development is focused on various aspects of time-domain investigation, such as variability detection, in particular via image-subtraction methods (Alard \& Lupton 1998; Bramich 2008), event classification (Bloom et al. 2012; Brink et al. 2013) and real-time processing and follow-up (Gal-Yam et al. 2011).

Finally, some of the most exciting frontiers, particularly those related to cosmic explosions, require wide-field time-domain imaging surveys. Examples include the discovery of rare or unusual transient events (Quimby et al. 2007; Barbary et al. 2009; Gal-Yam et al. 2009; Quimby et al. 2011; Gal-Yam 2012; Gezari et al. 2012; Chornock et al. 2013; Cenko et al. 2012, 2013), as well as systematic studies of unbiased object samples (Arcavi et al. 2010; Neill et al. 2011; van Velzen et al. 2011; Quimby et al. 2013; Gezari et al. 2013). An additional exciting prospect is the discovery and follow-up of EM counterparts of poorly localized non-EM signals, such as high-energy neutrino or gravitational-wave (GW) sources (Nakar \& Piran 2011; Metzger \& Berger 2012; LIGO Scientific Collaboration et al. 2012; Abbasi et al. 2012).

It should therefore not come as a surprise that the Large Synoptic Survey Telescope, a wide-field optical survey telescope, was the top choice of the US astronomical community for ground-based astronomy. Radio astronomers are developing powerful mapping machines such as LOFAR, MWA and APERTIF (and ultimately SKA) that will also study transients. The dynamic sky has been a major science driver for high-energy $\mathrm{X}$ - and $\gamma$-ray astronomy, with current (Swift, Fermi, MAXI/ISS) and future (e.g., AstroSAT, SVOM, LOFT) space missions offering wide-field capabilities.

In contrast, there has been little time domain study and exploration in the ultra-violet $(100-300 \mathrm{~nm})$. This is all the more surprising given that several major questions in astronomy (reviewed below) can be addressed even by a modest UV time domain explorer. Historically, exploration of the UV by photoelectric missions (OAO 2-3, TD-1A, ANS) provided photometry and spectroscopy of stars and studied bright Galactic variables and novae (Code et al. 1970; Rogerson et al. 1973). Subsequent missions such as IUE, FUSE, and the Hubble Space Telescope (HST) focused on single-object spectroscopy, with narrow fields of view prohibiting survey operations. Only in 2003 the $1.2 \mathrm{deg}^{2}$ field GALEX mission (Martin et al. 2005) begun the first systematic study of the extra-galactic static sky, and a more very limited time-domain program (Welsh et al. 2005; Gezari et al. 2013).

Below we give an overview of possible science that will be enabled by a wide-field UV transient explorer. Section 2 describes how the death of massive stars can be explored. 
In Section 3 we discuss gamma-ray burst (GRB) afterglows and orphan GRBs. In Section 4 we outline the study of tidaldisruption events (TDEs) and in Section 5 describe studies of active galactic nucleus (AGN) variability. Sections 6 and 7 discuss planetary transits and variable star studies respectively, while Section 8 discusses solar-system studies. In Section 9 we consider searches for the EM counterparts to gravitational-wave and high-energy neutrino sources. In Section 10 we describe the design parameters of the proposed ULtraviolet TRansient Astronomical SATellite (ULTRASAT) mission which we use as fiducial to quantify our predictions above. We conclude in Section 11 with a summary of the expected impact of an ULTRASAT-like mission.

\section{DEATH OF MASSIVE STARS}

The explosive death of massive stars as supernovae ( $\mathrm{SNe})$ is a complex unsolved astrophysical questions, defined as a science frontier question by the 2010 Decadal Survey. ${ }^{8}$ Determining the physical properties of massive stars prior to explosion is a critical step toward solving this problem; the pre-explosion stellar state sets the initial conditions to any computational investigation of the explosive process. Direct identification of SN progenitor stars in pre-explosion images is limited, as it can only be applied to explosions in nearby galaxies (typically $\sim 20 \mathrm{Mpc}$ away) and requires that high-spatial-resolution and deep images (mostly by $H S T$ were acquired prior to the explosion. To date, only about ten such relatively nearby massive stars have been confirmed as SN progenitors (Smartt et al. 2009; Maund et al. 2014).

Early UV observations of SN explosions provide a powerful method to study the properties (e.g., radius, surface composition) of exploding massive stars (Chevalier 1992; Matzner \& McKee 1999; Nakar \& Sari 2010; Rabinak \& Waxman 2011). Following the SN explosion a shock wave propagates outward from the core of the star through its optically thick envelope. When the shock wave reaches the outer regions where the optical depth is such that the photon diffusion time scale is shorter than the hydrodynamical time scale, the photons can escape the star; this is usually called the shock breakout flare, and would constitute the first EM signal for the explosion that an outside observer can detect. For supergiant stars the initial shock breakout signal is expected to be in the X-ray/UV and its duration is directly proportional to the radius of the progenitor star. Following this initial flare, the thermal energy deposited by the shock in the expanding envelope continues to diffuse out; we will call this the shock cooling emission. The bolometric luminosity of the shock cooling emission is almost constant, while the temperature of the radiating gas declines. The shock cooling signal will be prominent in the UV. The measured flux will rise as the peak of the emitted spectrum cools and passes though the observed band and will then decline as further cooling drives the emission peak to redder wavelengths (Figure 1, bottom). The rate of cooling (and thus the time it takes for the flux to peak in a given band) depends on the stellar radius and the composition of the envelope which determines the opacity. For supergiant star explosions with thick hydrogen envelopes, the opacity is known (Thomson scattering) and time independent, so the radius is straightforwardly inferred. For compact Wolf-Rayet (W-R) stars the opacity is time-dependent and a function of the surface composition (mass fraction of $\mathrm{He}, \mathrm{C}$ and O). Rabinak \& Waxman (2011) show that, given a well-sampled

\footnotetext{
8 New Worlds, New Horizons in Astronomy and Astrophysics, pp 57, 247, SSE-3.
}

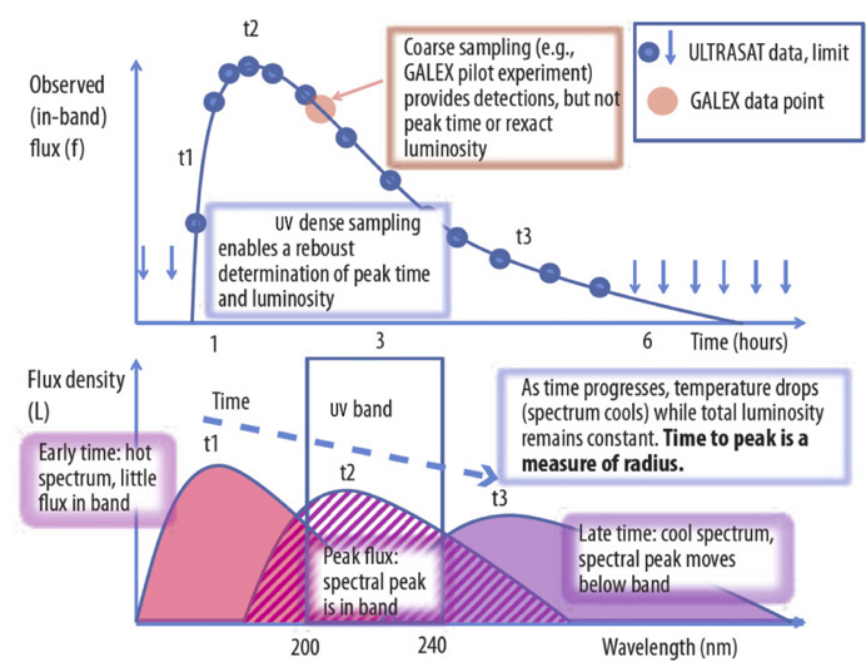

Figure 1. Bottom: a heuristic description of shock cooling emission from a massive star explosion. While the bolometric luminosity is almost constant, the decline in temperature leads to a rise and fall when observing in a given UV band. A well measured UV light curve (top) provides a measurement of the radius and surface composition of the exploding star. ULTRASAT is a proposed UV transient explorer-see Section 10.

(A color version of this figure is available in the online journal.)

light curve, one can infer both the stellar radius and the surface composition, as well as the ratio of explosion energy to ejected mass $(E / M)$ and the relative extinction toward an explosion.

The shock-cooling emission lasts a few hours for compact W-R stars (Rabinak \& Waxman 2011; Piro \& Nakar 2012) and approximately a day for red supergiants (Nakar \& Sari 2010; Rabinak \& Waxman 2011). This early emission provides constraints on the progenitor radius and chemical composition, which can not be derived from later ( $>$ few days) ground observations, since by the time the shock cools enough for visible light to be below the peak emissivity, complicating radiation from other sources (e.g., radioactivity, recombination) interferes with these measurements, and the total emission from shock cooling in compact stars become very faint and difficult to observe.

A UV wide-field transient explorer can detect the shock breakout flare from the largest stars, and those exploding within an extended circum-stellar medium (e.g., Ofek et al. 2010), as well as the shock cooling signal from numerous supergiant and $\mathrm{W}-\mathrm{R}$ massive stars, as predicted by theory and demonstrated by available observations (Figure 2). Combined space-UV and ground-based observations triggered by a UV transient explorer would yield an unprecedented wealth of data about massive star explosions (Table 1), going beyond the stellar radius and surface composition (and thus the stellar class of the progenitor: red or blue supergiant, or W-R star). Such information includes direct measurements of the dust extinction curve toward the progenitor location, removing uncertainties in measured quantities due to extinction (Rabinak \& Waxman 2011) and the amount of radioactive ${ }^{56} \mathrm{Ni}$ into the ejecta (a probe of the explosion mechanism and geometry; Piro \& Nakar 2012). As the early UV data measure the ratio of explosion energy to ejected mass, $E / M$, derivation of the ejecta mass $M$ from modeling of latetime data (light curves and nebular spectra) would provide information about the explosion energy. Measurements of earlyUV photometry and early spectroscopic velocity measurements that diverge from predictions of simple models would indicate non-standard stellar density profiles (e.g., Bersten et al. 2012) 
Table 1

Physical Properties Derived from Early UV Observations of Massive Star Explosions

\begin{tabular}{|c|c|c|}
\hline Physical Property & Required Data & References \\
\hline Pre-explosion stellar radius & Early UV photometry & $\begin{array}{l}\text { Nakar \& Sari (2010) } \\
\text { Rabinak \& Waxman (2011) }\end{array}$ \\
\hline Surface chemical composition & Early UV photometry & Rabinak \& Waxman (2011) \\
\hline $\begin{array}{l}\text { Dust extinction curve } \\
\text { toward the SN }\end{array}$ & $\begin{array}{l}\text { Early UV photometry } \\
\text { augmented by ground } \\
\text { optical/IR data }\end{array}$ & Rabinak \& Waxman (2011) \\
\hline $\begin{array}{l}\text { Amount of radioactive } \\
{ }^{56} \mathrm{Ni} \text { mixing into } \\
\text { the ejecta }\end{array}$ & $\begin{array}{l}\text { Early UV photometry } \\
\text { plus optical photometry } \\
\text { and early spectroscopy }\end{array}$ & Piro \& Nakar (2012) \\
\hline Explosion energy & $\begin{array}{l}\text { Early UV photometry } \\
\text { and late-time ground } \\
\text { observations }\end{array}$ & Rabinak \& Waxman (2011) \\
\hline Progenitor density profile & $\begin{array}{l}\text { Early UV photometry } \\
\text { augmented by spectroscopic } \\
\text { temperature measurements }\end{array}$ & Bersten et al. (2012) \\
\hline Recent progenitor mass-loss & $\begin{array}{l}\text { Early UV photometry } \\
\text { combined with spectroscopic } \\
\text { velocity measurements }\end{array}$ & $\begin{array}{l}\text { Ofek et al. (2010, 2013a, 2013b, 2013c, 2014) } \\
\text { Chevalier \& Irwin (2011) } \\
\text { Balberg \& Loeb (2011) } \\
\text { Svirski et al. (2012) }\end{array}$ \\
\hline
\end{tabular}

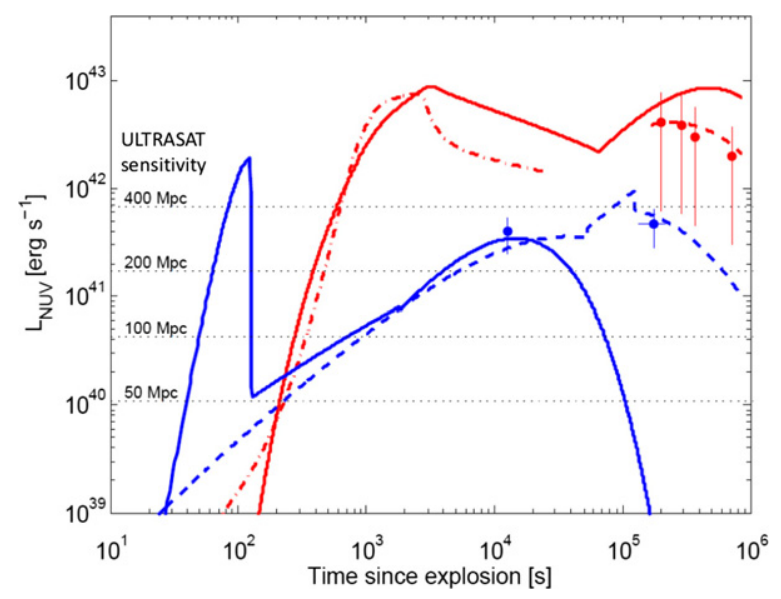

Figure 2. Theoretical shock-cooling models in the near UV band (200-240 nm) (solid: Nakar \& Sari 2010; dashed: Rabinak \& Waxman 2011; dash-dot: Sapir et al. 2013) compare well with observations (red data points: binned observations of Type II SNe from Schawinski et al. 2008 and Gezari et al. 2008b; blue points: binned observations of Type Ib SN 2008D from Soderberg et al. 2008). Red and blue curves are predictions for red supergiant and W-R star explosions, respectively.

(A color version of this figure is available in the online journal.)

while early UV observations of explosions occurring in thick circum-stellar medium would probe the final stages of massive star evolution just prior to explosion (e.g., Ofek et al. 2013c and references within). Assuming fiducial survey parameters (Section 10), the known UV signals (Figure 2) and local SN rates, one could study $\sim 100$ such events per year. ${ }^{9}$ Analysis of such a sample of massive star explosions would shed new light on the final stages of massive-star evolution and the explosive deaths of these stellar giants.

\footnotetext{
9 It is interesting to note that should a nearby SN Ia occur within the surveyed field of view, limits on the shock-cooling emission from it would place interesting constrains on the progenitor system (Kasen 2010; Piro et al. 2010; Rabinak et al. 2012).
}

\section{GRB AFTERGLOWS}

\subsection{Untriggered GRBs}

More than half of all GRBs are associated with an optical/UV counterpart signal that lasts between minutes to few days (Cenko et al. 2009). The afterglow is supposedly generated by the interaction of relativistic expanding shells with the surrounding medium. The resulting light curve is a complex, time-dependent combination of several components (reverse shock, jet break, density bumps, late energy injection) and unraveling them provides valuable information regarding the physics and energies of the explosion.

Detection of the afterglow in the near UV (NUV) is limited to the closest GRBs since above $z \sim 1.2$ host galaxy Lyman limit absorption will suppress the signal. We estimate the fraction of GRB afterglows that will be detectable in the UV at a fiducial sensitivity (Section 10) using the complete sample of Cenko et al. (2009) and typical afterglow power law: $F_{\nu} \propto v^{-\beta}$ with $\beta \approx 0.8$ (Sari et al. 1998). Of the $\sim 1000$ GRBs occurring every year, about 20 will happen within the field of view of a mission with fiducial parameters as specified in Section 10, approximately six will be in the redshift range such that their afterglow can be detected in the NUV (Jakobsson et al. 2012) and four will be brighter than NUV 21 mag AB (Nakar \& Piran 2003). These GRBs will therefore be observed regardless of a high-energy trigger, and their early afterglow emission will be followed continuously at minute-timescale temporal resolution.

\subsection{Orphan GRB}

GRBs are assumed to be collimated explosions, powered by ultra-relativistic jets that are a few degrees wide (see Piran 2004 for review). Although indirect evidence supports this model, a direct observational demonstration of the collimated nature of the outflow would be very valuable.

A testable prediction of the narrow jet model is that the radiation beaming angle should become wider with time as the jet decelerates. Thus, low-energy afterglow emission recorded hours-days after the burst should be seen by observers out of the 
initial opening angle of the prompt gamma-ray emission cone. The hypothesized event of afterglow emission seen without a high-energy (gamma- or X-ray) emission has been termed "orphan GRB" (Rhoads 1997).

The orphan events are 100 time more abundant (Guetta et al. 2005; Ghirlanda et al. 2013) but are orders of magnitude fainter than the GRB prompt emission and have not been detected so far, with a single possible exception (Cenko et al. 2013). In flux limited surveys we expect a $\sim 1: 1$ ratio of orphan to regular GRBs (Nakar \& Piran 2003). A wide-field UV transient explorer will thus be able to detect of order 10 events per year. Assuming future high-energy missions maintain the current sky coverage provided by Swift and Fermi/GBM ( $\sim 50 \%)$, we can expect a handful of bona-fide orphan afterglows per year, i.e., events which are detected as UV transients (with precise temporal information and spatial localization) and yet have no highenergy detection, even though they have occurred within sky areas covered by sensitive space missions. Later optical/radio observations would be useful to confirm the identity of such transients, e.g., by identification of an associated GRB-SN, or a long-lived radio afterglow (Cenko et al. 2013). Detection of even a single orphan afterglow will provide a valuable direct confirmation of the GRB jet model. The ratio of orphan GRB afterglows (prompt UV without high-energy emission) to normal events (prompt $\gamma$-ray emission and UV afterglow) will measure the GRB jet opening angle. A measurement of the average jet opening angle will allow translation of observed to isotropic energy, settling the true energy budget of GRBs.

\section{TIDAL DISRUPTION FLARES}

When a star passes close enough to a supermassive black hole $(\mathrm{SMBH})$ with a mass $<10^{8} M_{\odot}$ (for a solar mass main sequence star) it is shredded by tidal forces. Part of the stellar material goes into a bound orbit, creating a short-lived accretion disk around the $\mathrm{SMBH}$, leading to flaring emission in UV-X-ray light. Such TDEs, first considered by Lacy et al. (1982), are of extreme interest for the following reasons.

First, they are probing the properties of SMBHs in the center of inactive galaxies, which are difficult to observe by other means. Second, they probe the stellar population and dynamics at the vicinity of these black holes (BHs), which determines the rate at which the $\mathrm{SMBH}$ is fed. Third, they can provide a robust estimate of the rate of GWs from extreme mass ratio inspirals. Finally, we have still a lot to learn about the physical processes that are involved in the sequence of disruption, accretion disk build up, the accretion itself and the generation of various types of outflows, all reflected in the emission profile and spectrum of such events.

A large sample of observed TDEs would be extremely useful to tackle the above questions. For example, a study of the dependence of TDE rates and properties on the galaxy type can provide us with a completely new angle to study the connection between galaxy formation and the growth of SMBHs; the rates in particular are expected to be strongly dependent (and therefore an excellent probe) of the inner structure of galaxies. Triaxial galactic nuclei will lead to enhanced rates (by 1-2 orders of magnitude; Poon \& Merritt 2004) compared to standard spherical models (Magorrian \& Tremaine 1999).

To date there are only a handful of TDE candidates, observed in gamma-ray (Bloom et al. 2011; Burrows et al. 2011; Cenko et al. 2012) X-ray (Esquej et al. 2008; Cappelluti et al. 2009; Saxton et al. 2012), optical (van Velzen et al. 2011) and UV (Gezari et al. 2006, 2008a, 2012). None of these candidates is a confirmed TDE, mainly due to the difficulty of ruling out contamination by other object classes (mainly unusual $\mathrm{SNe}$ near galactic nuclei, and flares from AGNs that are "mostly dormant"). At least in one case the evidence seems strong. PS1-10jh (Gezari et al. 2012), detected by the Pan-Starrs ground-based optical survey and studied in the UV by GALEX, shows spectroscopic signatures of a helium-rich outflow, which may be interpreted as the disruption of a helium-rich stellar core; the spectrum does not match any known SN class, and is inconsistent with H-rich outflows from AGNs. However, the current small and non-uniform sample of candidates does not enable significant progress regarding the interesting open questions described above. For that purpose, a well-understood sample of dozens of observed TDEs is needed.

The UV is an optimal band to look for TDEs, since this is where a large fraction of the accretion luminosity is released. The UV emission is expected to peak on a time scale of 10-30 days at a luminosity of $3 \times 10^{42}$ to $3 \times 10^{43} \mathrm{erg} \mathrm{s}^{-1}$ (Strubbe \& Quataert 2009; Lodato \& Rossi 2011). Thus a mission with our fiducial parameters (Section 10) will be sensitive to fluxes predicted by these models for emission during the super-Eddington accretion-driven wind (Strubbe \& Quataert 2009) and from the accretion disk (Lodato \& Rossi 2011) out to $2 \mathrm{Gpc}$ and $0.5 \mathrm{Gpc}$, respectively. Adopting a TDE volumetric rate based on analytic (Magorrian \& Tremaine 1999; Wang $\&$ Merritt 2004) and $N$-body simulations (Brockamp et al. 2011) estimates of galactic TDE rates, and a local density of SMBHs from Marconi et al. (2004) and Hopkins et al. (2007) of $4 \times 10^{-7} \mathrm{Mpc}^{-3} \mathrm{yr}^{-1}$, the mission is expected to detect hundreds of TDEs per year.

An independent consistent estimate is obtained from the detection of the single robust TDE candidate (PS1-10jh; Gezari et al. 2012) during the GALEX time-domain survey (TDS; Gezari et al. 2013). The TDS survey covered $40 \mathrm{deg}^{2}$ for 6 months (three 2 month seasons) and found at least one event, leading to a rate of one event per $20 \mathrm{deg}^{2} \mathrm{yr}^{-1}$. As can be seen from Figure 15 in Gezari et al. 2013, PS1-10jh was not the only potential TDE candidate within the GALEX survey, even though it had the largest NUV flux increase. Many other events were detected as weaker flares (in the sense of fractional NUV flux increase). Some of those were further studied and turned out to be, for example, SNe. Others were not studied, or the studies turned out to be inconclusive. Thus, the rate calculated assuming a single TDE in the GALEX survey is a lower limit. Our fiducial mission has similar sensitivity and covers $\sim 700 \mathrm{deg}^{2} \mathrm{yr}^{-1}$ of extragalactic high-latitude sky, leading to an estimated detection rate of $\sim 35$ events per year using the above rate ( $>10$ events per year at $90 \%$ confidence).

It is interesting to note that the signature of Helium-rich TDEs is quite distinct, since the two most likely contaminants, namely AGN flares and $\mathrm{SNe}$, are not known to produce spectra with only He II lines. On the contrary, H-rich TDEs can be easily confused with AGN flares (showing broad Balmer lines) or Type II SNe. Since these H-rich flares are more likely to be missed, we speculate that is it likely that some of the unconfirmed TDE candidates from GALEX are indeed TDEs for which an AGN or SN nature could not be ruled out. Regardless of this issue, the existence of flares detected by GALEX in addition to PS1-1jh, as evident in Figure 15 of Gezari et al. (2013), indicates that the TDE rate calculated by assuming that this was the only TDE within that survey is a lower limit.

A mission with the parameters described in Section 10 will have significantly higher temporal resolution than the GALEX 


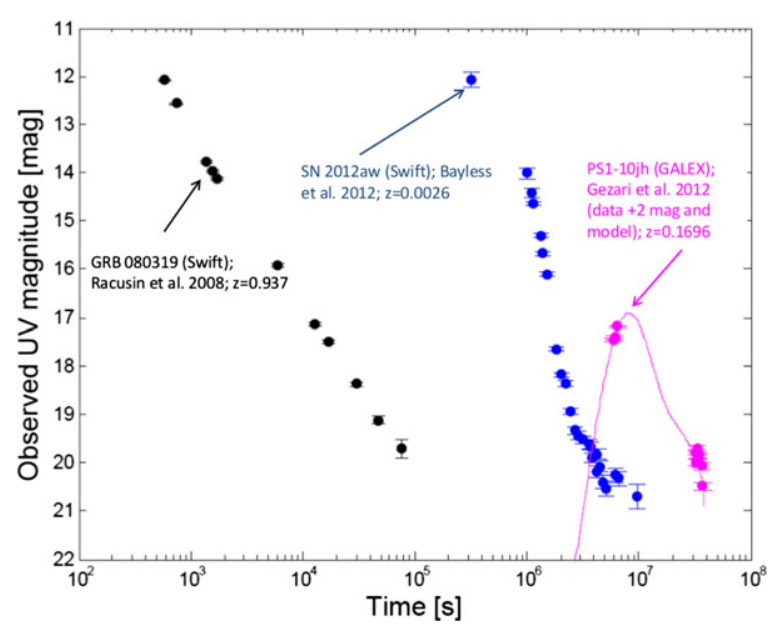

Figure 3. UV emission from supernovae, GRB afterglow and TDEs. Data are in approximately the mid-UV band (200-240 nm), and objects would be well above the sensitivity of our fiducial mission (GRB afterglow data from Racusin et al. 2008; SN 2012aw data from Bayless et al. 2013; TDE data and model from Gezari et al. 2012). Object classes are easily differentiated by their timescales (hours for GRBs, days for SNe, and months for TDEs). Supernovae will typically occur within relatively nearby, star-forming galaxies; GRBs occur in much more distant galaxies, while TDEs typically occur in more luminous hosts, and some would occur in early-type galaxies with little or no ongoing star formation. Note that a continuous sky monitoring by a wide-field UV explorer will provide early UV data, previously only available for GRB afterglows following high-energy triggers, also for SNe and TDEs.

TDS survey (2 days versus 12 minutes) and would therefore provide a clean separation from other transients (Figure 3). Detection of such a sample of TDEs will allow a systematic study of their properties and an examination of the rate as a function of host galaxy. Each detection will also place an upper limit on the SMBH mass, and thus probe correlations between host galaxy and $\mathrm{SMBH}$ masses (e.g., the $\mathrm{M}-\sigma$ relation).

\section{AGN VARIABILITY}

It has long been hoped that characterizing the multiwavelength continuum variability properties of the emission from massive BHs at the centers of galaxies (AGNs) will provide clues to the physical processes in the inner regions of their accretion disks, where most of the luminosity is produced. Over the past decade, this characterization has improved, based both on detailed many-epoch studies of samples with a few to about a hundred AGNs (Giveon et al. 1999; Kelly et al. 2009; Mushotzky et al. 2011), and on few-to-tens-of-epoch studies of samples with thousands of objects (e.g., Vanden Berk et al. 2004; Sesar et al. 2006; Wilhite et al. 2008; Welsh et al. 2011; Schmidt et al. 2012; MacLeod et al. 2012).

However, due to the limitations of both approaches, the picture is still unclear regarding the form and amplitude of the variability power spectrum, its dependence on physical parameters (redshift, luminosity, BH mass, Eddington ratio), and the correlations between variations in different wavelength bands (X-ray, UV, optical). Based on studies of a handful of Seyfert galaxies with good sampling, the power spectrum can be described as several broken power laws, with break frequencies that may scale inversely with $\mathrm{BH}$ mass, analogous to results for stellar-mass accreting BHs. Mushotzky et al. (2011) have recently used data for four Seyfert galaxies in the Kepler field to probe accurately, for the first time in the optical range, timescales as short as one month to a day. They found a significant

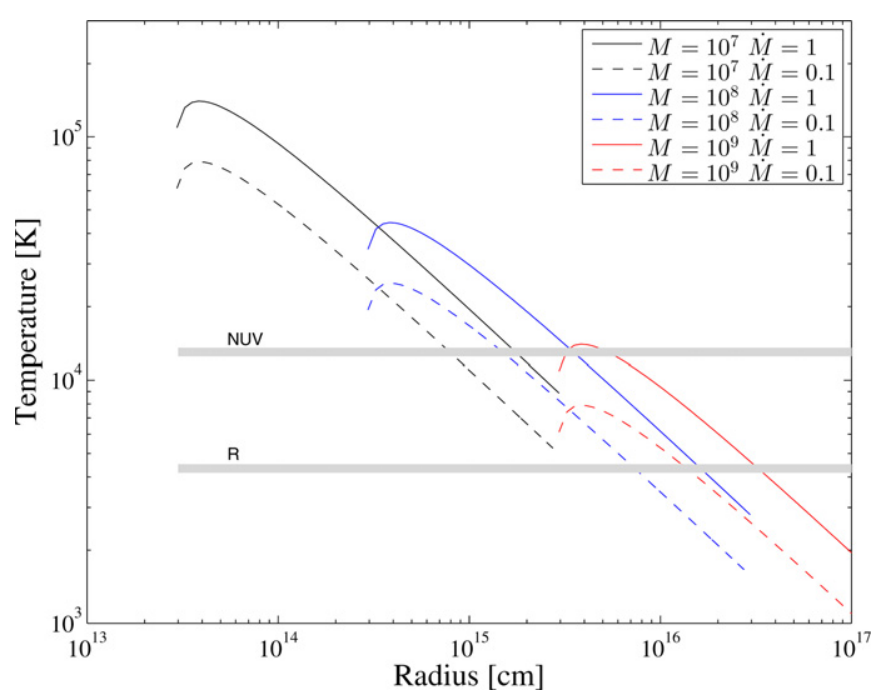

Figure 4. Temperature as a function of radius in a simple, optically thick, thin accretion disk for several black hole mass (marked by $M$ in units of $M_{\odot}$ ) and accretion rate (marked by $\dot{M}$ in units of $M_{\odot} \mathrm{yr}^{-1}$ ) values. The disk is assumed to span between 10 and 1000 Schwarzschild radii. The gray lines shows the temperature probed by the NUV and $R$ bands. The UV probes significantly smaller radii.

steepening of the power spectrum on these timescales, steeper than ever seen for AGNs either in the optical or in X-rays.

Figure 4 shows that observing in the NUV probes the inner regions of the accretion disk; NUV-band observations are sensitive to radii which are almost an order of magnitude smaller compared to those probed by $R$-band data. This makes a UV study complementary to large optical surveys, and yet opens a window into a relative unexplored region of the accretion disk around AGNs.

A UV transient explorer that would combine a wide field of view with minute-scale cadence will chart previously unexplored territory in quasar variability, obtaining both large samples of quasars, and many epochs per quasar. With a full month of continuous minute-scale exposures per field, the powerspectrum can be measured down to short timescales similarly to Mushotzky et al. (2011) but, rather than for four AGNs, for a significantly larger number of quasars (Figure 5).

Our estimate of the expected areal densities of QSOs for our fiducial survey parameters (Section 10), are based on the UV properties of a large number of SDSS QSOs observed in the UV by GALEX. We begin from the SDSS DR7 Quasar Cata$\log$ (Schneider et al. 2010), listing 105,783 spectroscopically confirmed, optically selected, un-obscured (i.e., broad-line or "type-I") AGNs, most of which are $i<19.1$ mag sources at $z<2$, selected over an effective area of $9380 \mathrm{deg}^{2}$. We then used the cross-matched GALEX/GR5-SDSS/DR7 catalog of Bianchi et al. (2011), to obtain NUV magnitudes (and errors) for all the sources. We used data obtained from all GALEX surveys, and limit our analysis to sources with photometric errors $\Delta \mathrm{NUV}<0.3 \mathrm{mag}$.

Figure 5 presents the cumulative areal density of SDSS quasars, according to their GALEX NUV fluxes. At our fiducial flux limit per single visit (NUV $=21$ ), we estimate a density of $>4.5 \mathrm{deg}^{-2}$. Assuming the fiducial field of view and yearly observing cycle of $\sim 10$ independent extragalactic footprints, the expected number of AGNs surveyed for variability is $\sim 36,000$.

We note that this is a lower limit, due the combination of several limitations of the data we used. First, the SDSS QSO catalog is naturally flux-limited, and thus does not contain 


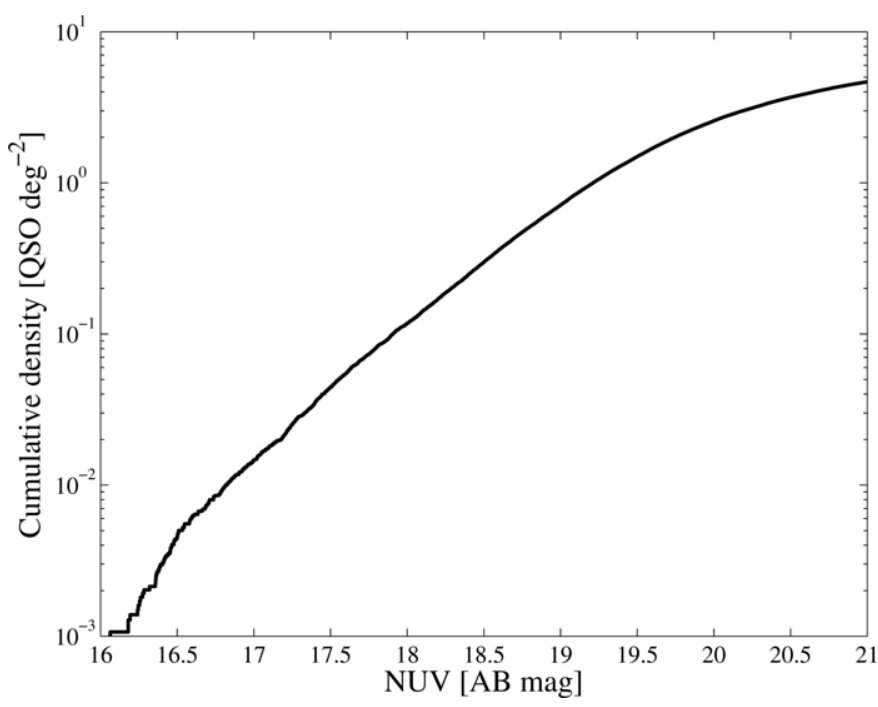

Figure 5. Cumulative areal density of spectroscopically confirmed, optically selected and NUV-detected QSOs, as revealed by cross-matching the SDSS/ DR7 QSO catalog of Schneider et al. (2010) and the GALEX/GR5 catalog. We only include sources with $\triangle \mathrm{NUV}<0.3 \mathrm{mag}$. This density is a lower limit on the areal density of QSOs in the NUV band.

(optically) faint QSOs, which might still be detected in the UV. Second, the GALEX catalog we used (GR5) includes mainly the rather shallow All Sky Imaging Surveys; the fiducial survey we consider here will be significantly deeper once coadded data are considered, and will thus include more UV-faint AGNs. In this context, it should be noted that the cumulative areal density of the spectroscopically confirmed SDSS QSOs alone (not shown here) reaches $\simeq 10 \mathrm{deg}^{-2}$.

Such a UV explorer mission would cover the poorly studied $\mathrm{UV}$, and rest-frame far UV for the higher- $z$ quasars (rather than the optical, where variations are smaller; e.g., Giveon et al. 1999; Welsh et al. 2011; Schmidt et al. 2012). Finally it will study bona-fide luminous quasars (which may possibly have lower variation amplitudes than the Seyferts, but this is basically unknown for these timescales). The sub-percent photometric precision for measuring comparable variation amplitudes on one-day timescales (as seen by Mushotzky et al. 2011) can be achieved by integrating many (>100) images with lower precision over a day (and further benefit will be provided by time dilation of high-redshift sources). The large sample will allow to bin objects according to the physical attributes derived from their spectra (e.g., BH mass and Eddington ratio) and inspect variability properties within each bin.

A UV transient explorer therefore has the potential for important new discoveries in the variability at short timescales in the UV for tens of thousands of luminous AGNs. Results would need to be reproduced by any successful model of AGN accretion disk physics. Apart from studying AGN physics, characterizing fast UV AGN variability would be important for filtering out false transient alerts by other large surveys searching for transient signals (e.g., Gal-Yam et al. 2002; MacLeod et al. 2012), as well as for producing variability-selected AGN samples for cosmological experiments like BigBOSS (Sholl et al. 2012).

\section{EXOPLANET TRANSITS}

Recent years have ushered a revolutionary advance in exoplanet detection. To date, several hundred stars have been con- firmed to host orbiting planets (Howard et al. 2012), the majority detected by their transit light curve (Bordé 2003) in visible light. Discovery of transiting planets relies on the detection of a deficit in the photon flux during the eclipse, a signal whose magnitude scales with the square ratio of the planetary to stellar radii, the eclipse duration, and the observation time (here assumed to be 30 days per field of view; Section 10). For a significant detection the flux deficit must exceeds a threshold noise level. A widefield UV transient explorer offers opportunities to detect planets. As quantified below, planetary transit detections are challenging and require precision measurements, so a conservative approach is warranted in evaluating the potential for such detections.

Several possible subclasses of sources are of particular interest. First, we consider planets orbiting UV-bright stars, particularly those orbiting $\mathrm{O}, \mathrm{B}$, and A type stars, for which no extensive survey has been performed (Johnson et al. 2011), and whose radiative envelopes are likely to be photometrically quiet (Simon et al. 2002). However, we note that the exact level of UV activity of early type stars is poorly explored. Since these types of stars are considerably shorter lived than Sun-like stars, such detections will provide a snapshot of solar systems in their early stages of formation (e.g., before or during planet migration), and probe planet formation around massive stars.

We estimate the potential results from a mission with our fiducial parameters (Section 10). We model factors contributing to the noise including background $\left(0.1\right.$ photons $\mathrm{s}^{-1}$ pixel $\left.^{-1}\right)$, dark current ( 0.05 electrons $\mathrm{s}^{-1}$ pixel $^{-1}$ ), readout (4 electrons per readout), digitization noise, shot noise (with a Poisson distribution), and an additional relative accuracy term. The latter accounts for errors induced by spatial variabilities in the detector and temporal variabilities of the starlight. Flat-field errors that vary among observations, variable background sources, multiple source confusion and associated jitter errors will all contribute uncertainty and reduce the effective photometric accuracy. Since some of these factors are still unknown, we consider the number of planets that may be found as a function of this additional noise.

We estimate the expected number of stellar sources in the field of view using the Besancon galactic model (Robin et al. 2003), corrected for the NUV observational band (200-240 nm) using mean spectra of known stars (Figure 6).

Taking stellar variability into account, we find that within each field of view (observed for 30 days by the nominal system) there are $\sim 1000$ stars for which our accuracy is sufficient to detect a transiting close-in Jupiter-size planet (Figure 7). With an estimated Jupiter-like planet abundance of $1 \%$ and a relative accuracy term of $10^{-3}$, the survey will find hundreds of such planets within the mission lifetime. It is still to be seen if relative accuracy term of $10^{-3}$ is realistic. Hot stars $(O, B$, and A type) constitute a significant fraction of these systems, such that detection (or non-detection), would constrain the planetary abundance around massive stars, in contrast with that of the older systems enumerated by Kepler. The radial velocity signal of planets orbiting hot stars is difficult to detect for verification. However, even a few detections will be important because of the young, relatively well defined age of such systems.

Secondly, white dwarfs (WDs) are rare and small, making the detection of orbiting planets challenging. However they are bright in the UV, and because of their small size, even relatively small planets can obscure a detectable fraction of the stellar light. While no such systems have been reported to date, a large UV sky survey will include more than 20,000 WD and may 


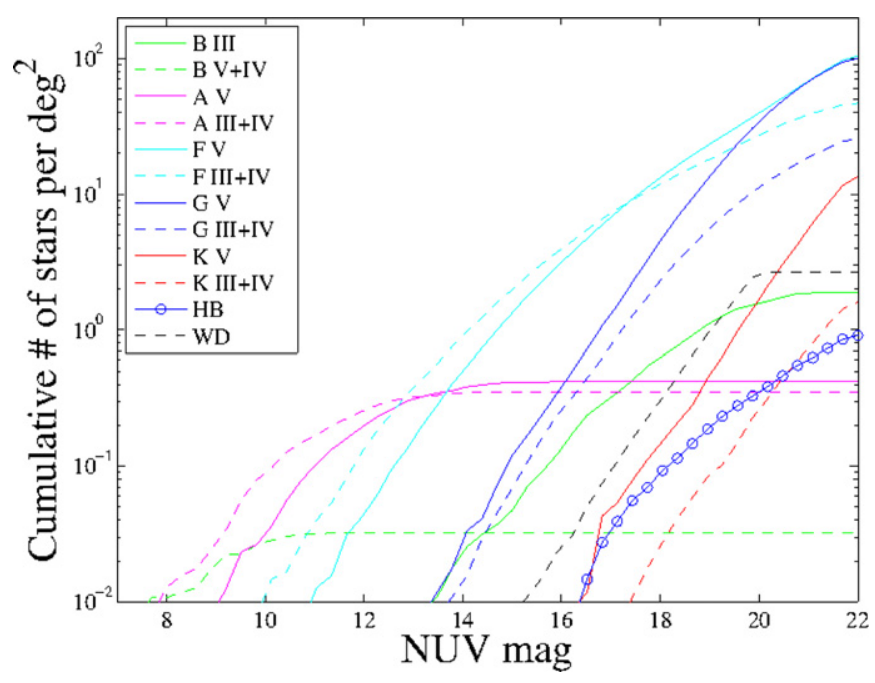

Figure 6. Cumulative number of stars of various types in the NUV band. These numbers are from a simulated catalog produced using the Besancon model (Robin et al. 2003). The B V and B IV type stars have similar contributions to the B V+B IV line. HB are horizontal branch stars.

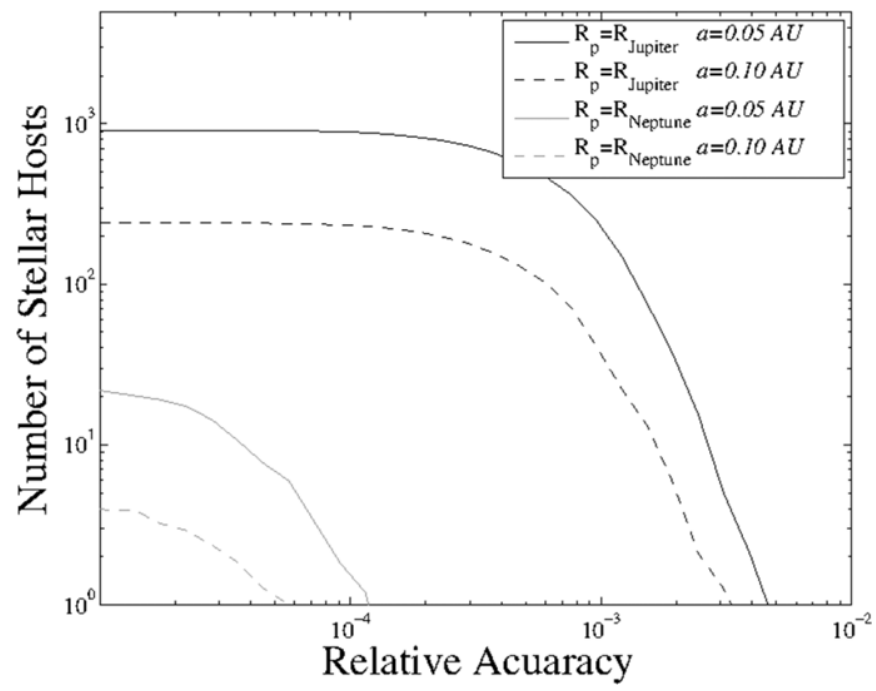

Figure 7. Number of stellar sources around which planets would be detectable by a wide-field UV transient explorer (if they exist), as a function of the system relative accuracy (i.e., additional non-Poisson noise). Jupiter-size planets would be detectable orbiting several thousands of stars in tight orbits $(a=0.05 \mathrm{AU})$, assuming an isotropic distribution of inclinations. This number decreases with distance from the star, and with planetary radius. Based on experience from ground-based wide-field experiments using CCD detectors (e.g., PTF; Ofek et al. 2012; van Eyken et al. 2011, 2012), we estimate a fiducial mission could achieve a relative accuracy better than $10^{-3}$. With an estimated hot Jupiter abundance of $1 \%$, such a survey will find hundreds of planets within the mission time.

enable the first such discovery (or set a limit on the planetary abundance around WDs).

\section{VARIABLE STARS}

Many types of variable stars have UV amplitudes which are larger than their visible light variations. Therefore, the UV band may shed new light on some types of variable stars. Here we discuss a few of these classes.

\subsection{Eclipsing Binaries}

Eclipsing binaries are important for our understanding of stellar parameters and the distance scale. Observations of these

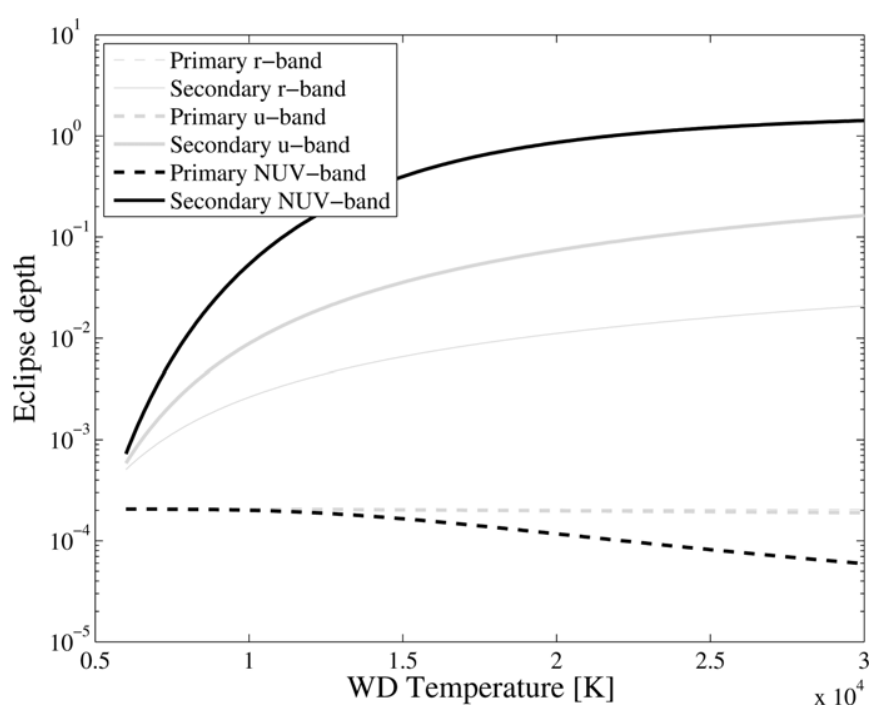

Figure 8. Expected primary (solid) and secondary (dashed) eclipse depths for a putative binary system with a main sequence $\mathrm{G}$ dwarf primary and a secondary white dwarf of varying temperature. One can see that the secondary eclipse is very prominent in the NUV compared to visible light, more so by orders of magnitude for hot white dwarf stars. UV observations can thus reveal an important population of hot WDs in multiple systems with main-sequence stars.

stars in the UV may provide important contributions. For most stars the NUV band is found at the Wein tail of their spectrum, and the luminosity in this band is very sensitive to temperature and metallicity. Therefore, NUV observations, along with visible-light observations, can provide a good description of the components in an eclipsing system, and in some cases can be used to identify systems that are extremely hard to detect in visible light. For example, consider a hot WD eclipsing a main sequence $\mathrm{G}$ star. In this case, both primary and secondary eclipse signals will have small amplitudes in visible light. However, the secondary eclipses will be quite prominent in the NUV band. Figure 8 shows the primary and secondary eclipse depth as a function of the WD temperature, in the $r, u$ and NUV-bands, assuming a primary star with a black body spectrum with temperature of $5700 \mathrm{~K}$ and one solar radius, and a WD radius of $10^{9} \mathrm{~cm}$. One can see that such a system will be difficult to detect in visible light; but the secondary eclipse signal will be orders of magnitude stronger in the NUV. Such main sequence WD systems (including binaries and triples with two WDs in a tight orbit) are of great interest, e.g., as putative progenitors of Type Ia SNe (Iben \& Tutukov 1984; Katz \& Dong 2012; Kushnir et al. 2013).

\subsection{RR Lyr Stars}

Having long served as Galactic distance indicators, RR Lyr stars have recently become a focus of additional scientific interest as popular tracers of Galactic halo structures (e.g., Sesar et al. 2010) and are being actively used to search for Galactic streams (e.g., Sesar et al. 2012; Drake et al. 2013). As the NUV band is in the Wein tail of the spectrum, the NUV variability amplitude of RR Lyr stars is large compared to the optical variability, making these stars more likely to be detected and their period measured. In addition, UV observations of RR Lyr stars present an opportunity to measure their surface temperature as a function of their phase (e.g., Wheatley et al. 2012). Moreover, Wheatley et al. (2012) showed that when the effective temperature of RR Lyr stars is at its minimum, NUV observations can be used to measure the metallicity of 
these stars. Such metallicity measurements would be especially valuable for faint and distant RR Lyr stars that trace ancient Galactic halo structure, providing a new key to the formation of our Galaxy.

\subsection{Cataclysmic Variables}

Cataclysmic variables (CV) are WDs accreting matter from a binary companions (Hellier 2001). Several sub-divisions of $\mathrm{CVs}$ are recognized, but a ubiquitous property of this class is that these systems emit strong episodic flares; these flare are typically very blue, and could manifest as many-magnitude flares in the blue and UV bands. The combination of timescales (days), flare amplitudes (magnitudes) and blue colors of the quiescent counterpart are quite unique to these systems.

One of the main observational gaps in studies of these systems is the scarcity of detailed temporal flare profiles; this is due to the fact that the flares are short, and systems are discovered in flare states by low-cadence surveys; in most cases the flare is already declining by the time detailed observations begin.

The fiducial mission described in Section 10 would be a great CV discovery machine, with its very wide field of view and NUV sensitivity, especially during the months that its orbit drives the field of view across the Galactic plane. The observing mode (almost constant monitoring) would provide, for the first time, detailed light curves of CV flares at minute time scales, probably resolving the rise and fine temporal structure (e.g., periodicity; flickering). In addition, alerts by such a mission would allow follow-up studies in other wavelengths (e.g., prompt X-ray studies with Swift or a successor mission). Such observations offer a broad potential for progress in deciphering the complex physics governing the various classes of CVs.

\subsection{Superflares on Solar Twins and Habitability}

The fact that the Sun is a relatively quiet star has a benevolent impact on life on Earth (and more recently, on modern technology and space travel). While many stars in the galaxy are known to episodically emit flares that are much more powerful (up to $\left.10^{39} \mathrm{erg}\right)$ compared with the strongest solar flares $\left(\lesssim 10^{32} \mathrm{erg}\right.$; Schaefer 2012 and references therein), it was often believed that solar twins (i.e., slowly rotating $\mathrm{G}$ dwarfs) are as quiet as the Sun.

However, it has recently been demonstrated using Kepler data (Maehara et al. 2012) that even solar twins have superflares that are orders of magnitude stronger than the strongest solar flares on record. As these flares have much better contrast in the NUV (flare flux relative to total photospheric stellar flux) they would be much easier to detect by a mission similar to our fiducial mission described below (Section 10). A broad census of the energy distribution and frequency of strong flares as a function of stellar type and other parameters (e.g., rotation), which such a mission would provide almost by a default, would be extremely valuable to understand flare physics, as well as any astrobiological implications. The potential to trigger multiwavelength follow-up of powerful flares from nearby solar twins is an especially attractive prospect.

\section{SOLAR SYSTEM OBJECTS}

Solar system objects are expected to be relatively faint in the NUV band. The reasons are that the solar NUV flux is low and that the albedo of asteroids in the NUV band is lower by a factor of two relative to their visible light albedo (Stern et al. 2011). Nevertheless, a wide-field UV mission will detect a wealth of asteroids and near Earth objects (NEOs). The colors can provide information about their surface composition, while their magnitude as a function of phase angle can provide information about their surface properties (through the opposition effect which is more prominent in the bluer bands; Hapke et al. 1998). Finally, continuous monitoring of asteroids on 10 minute to 30 day time scales, is likely to provide the best sample of asteroid rotation periods (e.g., Polishook et al. 2012).

Based on a simulations of the known asteroids in the solar system, assuming asteroids have a solar-like spectrum and NUV albedo which is half of the visible light albedo, a fiducial mission (Section 10) can detect about 60 asteroids in each field of view, of which $\sim 0.5$ are NEOs. Over three years baseline, such a mission can observe over 2600 unique asteroids of which 280 are NEOs.

Given the fiducial cadence (Section 10), we expect to measure the rotation periods for almost all of these asteroids. Observing solar system objects near opposition, the mission will generate accurate UV light curves of the opposition effect. This in turn will allow us to study, in a uniform way, the surface properties of a large sample of asteroids.

\section{GRAVITATIONAL WAVES AND HIGH-ENERGY $v$ COUNTERPARTS}

Experiments searching for GWs from astrophysical sources, such as Advanced LIGO (Harry \& LIGO Scientific Collaboration 2010), Virgo (Acernese et al. 2005) and LCGT (Kuroda et al. 2003) are expected to be operational in the near future. The best candidates predicted to produce detectable GW signals are the in-spiral events of double neutron stars (NS-NS) and neutron-star-black-hole (NS-BH) binary systems. Correlating a GW signal from these experiments with an EM counterpart will provide an independent verification and will allow detection of weak signals and therefore substantially improve the surveys effective sensitivity. In addition, the fine spatial localization afforded by EM counterparts would provide crucial information about the sources: measured redshifts of host galaxies would reveal the distance and thus the energy scale, while environmental information (e.g., young versus old galaxies) will shed further light on the physical nature of the emitting systems.

Several possible candidate EM counterparts to GW sources have been identified. These include short-duration gammaray bursts; IR/optical/UV "kilonova"/“macronova" (Kulkarni 2005; Metzger \& Berger 2012) and radio afterglows (Nakar \& Piran 2011). GW signal localizations will be, at best, down to tens of square degrees (Nissanke et al. 2011). Telescopes with exceptionally wide fields of view will be required to search such large areas. One of the major strength of a wide-field space-UV mission would be its capability to rapidly search and identify such EM counterparts and pinpoint their astrophysical source, if they are UV luminous. Both operational directions-a transient detection in the UV of a potential "macronova" signal which will be followed by a search in GW detector data, or a GW signal that will trigger a target-of-opportunity (ToO) for the satellite mission-are valuable options.

The estimated GW detection rate, with large uncertainties, is $40 \mathrm{yr}^{-1}$ for NS-NS (Abadie et al. 2010; Kopparapu et al. 2008) and $10 \mathrm{yr}^{-1}$ for NS-BH. Most GW sources that can be detected at reasonable signal-to-noise ratios by the above experiments will originate from a distance $<50 \mathrm{Mpc}$, close enough to possibly provide good signal-to-noise ratio in the UV for a mission with fiducial parameters given in Section 10 (down to events $\sim 10$ times less luminous than typical SN flares). 


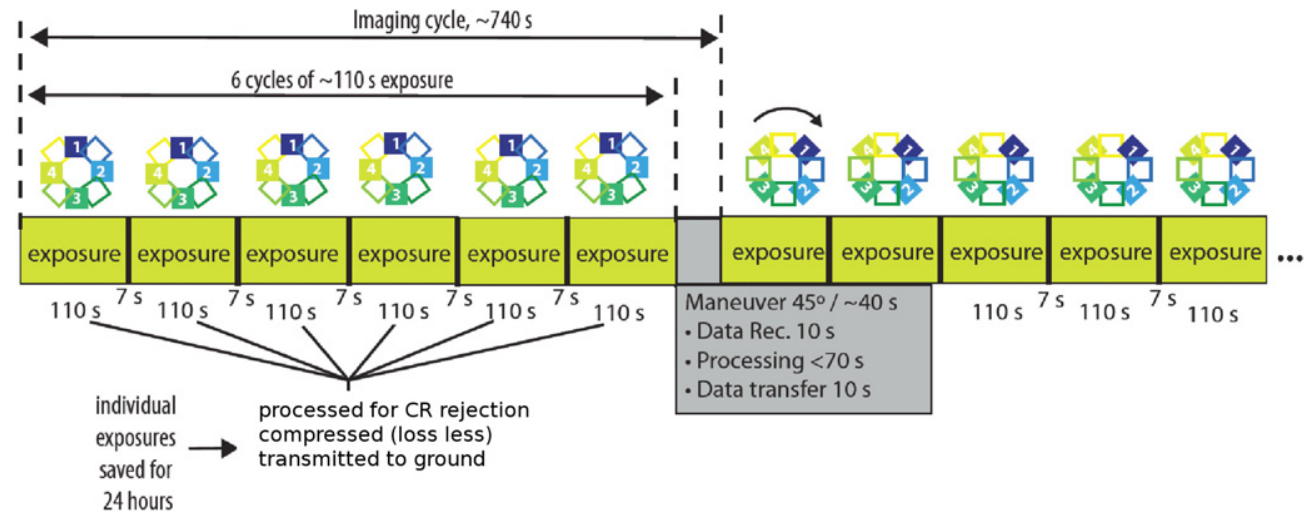

Figure 9. Spatial locations of the alternating ULTRASAT field of view. Each image includes six $110 \mathrm{~s}$ exposures where the orientation is fixed. The four telescopes are shifted by 45 deg every $660 \mathrm{~s}$.

(A color version of this figure is available in the online journal.)

Table 2

ULTRASAT Mission Design Parameters

\begin{tabular}{ll}
\hline \hline Parameter & \multicolumn{1}{c}{ Value } \\
\hline Field of view & $802 \mathrm{deg}^{2}$ \\
Cadence & 12 minutes \\
Spatial resolution & $19^{\prime \prime} .3$ \\
Wavelength band & $200-240 \mathrm{~nm}$ \\
Limiting mag & $21(5 \sigma$ detection; 12 minute coadd $)$ \\
Focal length & $f 2.4$ \\
Aperture & $13.3 \mathrm{~cm}$ \\
\hline
\end{tabular}

A wide-field transient explorer may significantly enhance also the sensitivity of high-energy neutrino detectors (Halzen 2007; Montaruli 2012) such as IceCube by reducing their backgrounds through precise timing of the SN explosion providing coincidence with an otherwise sub-threshold neutrino signal. Potential sources include SN explosions with failed or "choked" jets, that accelerate particles to high energies (leading to pion and neutrino emission) but fail to puncture the stellar surface. Such explosions may be quite common (e.g., appearing as normal $\mathrm{SNe} \mathrm{Ib} / \mathrm{c}$; Razzaque et al. 2004). Discovery of even a single case would be a breakthrough in high-energy neutrino research, as well as in understanding SN explosions. Pinpointing an astrophysical source of neutrinos will shed light also on the related open question of the origin of ultrahigh energy cosmic-rays (Waxman 2011), and will enable probing fundamental neutrino properties (e.g., flavor oscillations and coupling to gravity) with an accuracy many orders of magnitude beyond what is currently possible, e.g., using upward moving $\tau$ particles (Waxman \& Bahcall 1997).

\section{A FIDUCIAL MISSION DESIGN: THE ULTRASAT MISSION}

ULTRASAT is a proposed wide-field transient explorer satellite mission. The general parameters of the instrument are given in Table 2.

The instrument includes an array of four identical UV imaging $13.3 \mathrm{~cm}$ aperture refractive telescopes $(f / 2.4)$, each with a field of view of $11 \mathrm{deg} \times 11 \mathrm{deg}$. A UV enhanced $\delta$-doped (Nikzad et al. 2011) $4 \mathrm{k} \times 4 \mathrm{k} 15 \mu \mathrm{m}$ pixel quad-readout CCD will be mounted at the focal plane of each telescope, providing a pixel scale of 19 '. 3 pixel $^{-1}$ after $2 \times 2$ binning. Reflective filters are used to prevent out-of-band light from reaching the CCD detectors. The spatial resolution is more than sufficient to determine the position of transient sources to within an individual typical galaxy. The telescopes always observe roughly pointing at the anti-Sun direction. The satellite rotates about the field of view axis eight times in an orbit in order to maintain its orientation with respect to the Earth. The telescopes are arranged such that after a $45^{\circ}$ rotation alternate fields are interleaved with some overlap, and following an additional $45^{\circ}$ rotation, the original field is revisited (Figure 9). The total sky coverage is $802 \mathrm{deg}^{2}$ (sampled at alternating steps) with $166 \mathrm{deg}^{2}$ overlap observed at every orientation. Each sky position is thus sampled at a cadence between $110 \mathrm{~s}$ to 24 minutes during approximately one month, as the entire field of view drifts across the sky due to the satellite Sun-synchronous orbit.

Each image will be composed of six separate exposures of $\sim 110 \mathrm{~s}$ stored in memory and co-added after cosmic ray rejection. During each image $(\sim 12$ minutes including readout time) the satellite will maintain its pointing and orientation with sub-pixel stability. The images will then be compressed and transmitted to the ground. In addition, $24 \mathrm{hr}$ of raw individual exposures are stored to provide, on request, higher temporal resolution for specific events (cutouts). The satellite will be placed at a low Earth orbit with an inclination of $82^{\circ}$ allowing unobstructed continuous observations of a region in the direction opposite the Sun. As the Earth revolves around the Sun the telescope field of view will shift ( $\sim$ once a day), keeping it centered at the anti-Sun direction and keeping a maximal margin from the Earth, to minimize the effects of stray light and earthshine. The satellite will be equipped with a realtime communication system based on commercial geostationary communication satellites. The communication system will have sufficient bandwidth to transmit full images (compressed) to the ground. The images will be processed within 10 minutes of arrival to identify transient signals and alerts will distributed for follow-up by telescopes world wide. In addition ULTRASAT will have the capability to receive ToO alerts. Considering orbit and pointing limitations the telescopes will have the ability to point at a given $\mathrm{ToO}$ within 30 minutes. More details are available at the project Web site. ${ }^{10}$

\section{CONCLUSIONS}

We have reviewed the potential science impact of a widefield UV transient explorer. In particular, we consider studies of massive star explosions, GRBs and TDEs (Figures 2 and 3),

\footnotetext{
10 http://www.weizmann.ac.il/astrophysics/ultrasat/
} 
as well as variability studies of AGNs, planetary transits and various classes of variable stars. Studies of EM counterparts to GW or high-energy $v$ sources also offer an exciting prospect. We quantify our analysis using fiducial parameters similar to those of the proposed ULTRASAT mission, and show that such a mission would have a strong impact on studies of massive stellar death, GRBs and TDEs, as well as numerous other subjects including extra-solar planets. It appears that rich scientific returns can be obtained using a modest space mission focused on wide-field UV transient surveys.

We thank M. Van Kerkwijk and B. E. Schaefer for useful discussions. This research has been supported by grants from the Israeli Space Agency and the Keck Institute for Space Science (KISS).

\section{REFERENCES}

Abadie, J., Abbott, B. P., Abbott, R., et al. 2010, CQGra, 27, 173001 Abbasi, R., Abdou, Y., Abu-Zayyad, T., et al. 2012, A\&A, 539, A60 Acernese, F., Amico, P., Al-Shourbagy, M., et al. 2005, CQGra, 22, 869 Alard, C., \& Lupton, R. H. 1998, ApJ, 503, 325

Arcavi, I., Gal-Yam, A., Kasliwal, M. M., et al. 2010, ApJ, 721, 777 Balberg, S., \& Loeb, A. 2011, MNRAS, 414, 1715

Barbary, K., Dawson, K. S., Tokita, K., et al. 2009, ApJ, 690, 1358 Bayless, A. J., Pritchard, T. A., Roming, P. W. A., et al. 2013, ApJL, 764, L13 Becker, R. H., White, R. L., \& Helfand, D. J. 1995, ApJ, 450, 559 Bersten, M. C., Benvenuto, O. G., Nomoto, K., et al. 2012, ApJ, 757, 31 Bianchi, L., Efremova, B., Herald, J., et al. 2011, MNRAS, 411, 2770 Bloom, J. S., Giannios, D., Metzger, B. D., et al. 2011, Sci, 333, 203 Bloom, J. S., Richards, J. W., Nugent, P. E., et al. 2012, PASP, 124, 1175 Bordé, P. 2003, PhD thesis, Universit Pierre \& Marie Curie (Paris 6) LESIA (UMR 8109), Observatoire de Paris, France

Bramich, D. M. 2008, MNRAS, 386, L77

Brink, H., Richards, J. W., Poznanski, D., et al. 2013, MNRAS, 435, 1047 Brockamp, M., Baumgardt, H., \& Kroupa, P. 2011, MNRAS, 418, 1308 Burrows, D. N., Kennea, J. A., Ghisellini, G., et al. 2011, Natur, 476, 421 Cappelluti, N., Ajello, M., Rebusco, P., et al. 2009, A\&A, 495, L9 Cenko, S. B., Kelemen, J., Harrison, F. A., et al. 2009, ApJ, 693, 1484 Cenko, S. B., Krimm, H. A., Horesh, A., et al. 2012, ApJ, 753, 77

Cenko, S. B., Kulkarni, S. R., Horesh, A., et al. 2013, ApJ, 769, 130 Chevalier, R. A. 1992, ApJ, 394, 599

Chevalier, R. A., \& Irwin, C. M. 2011, ApJL, 729, L6

Chornock, R., Berger, E., Rest, A., et al. 2013, ApJ, 767, 162

Code, A. D., Houck, T. E., McNall, J. F., Bless, R. C., \& Lillie, C. F. 1970, ApJ, 161,377

Condon, J. J., Cotton, W. D., Greisen, E. W., et al. 1998, AJ, 115, 1693

Drake, A. J., Catelan, M., Djorgovski, S. G., et al. 2013, ApJ, 765, 154

Esquej, P., Saxton, R. D., Komossa, S., et al. 2008, A\&A, 489, 543

Gal-Yam, A. 2012, Sci, 337, 927

Gal-Yam, A., Kasliwal, M. M., Arcavi, I., et al. 2011, ApJ, 736, 159

Gal-Yam, A., Mazzali, P., Ofek, E. O., et al. 2009, Natur, 462, 624

Gal-Yam, A., Ofek, E. O., Filippenko, A. V., Chornock, R., \& Li, W. 2002, PASP, 114,587

Gezari, S., Basa, S., Martin, D. C., et al. 2008a, ApJ, 676, 944

Gezari, S., Chornock, R., Rest, A., et al. 2012, Natur, 485, 217

Gezari, S., Dessart, L., Basa, S., et al. 2008b, ApJL, 683, L131

Gezari, S., Martin, D. C., Forster, K., et al. 2013, ApJ, 766, 60

Gezari, S., Martin, D. C., Milliard, B., et al. 2006, ApJL, 653, L25

Ghirlanda, G., Ghisellini, G., Salvaterra, R., et al. 2013, MNRAS, 428, 1410

Giveon, U., Maoz, D., Kaspi, S., Netzer, H., \& Smith, P. S. 1999, MNRAS, 306, 637

Guetta, D., Piran, T., \& Waxman, E. 2005, ApJ, 619, 412

Halzen, F. 2007, Sci, 315, 66

Hapke, B., Nelson, R., \& Smythe, W. 1998, Icar, 133, 89

Harry, G. M., \& LIGO Scientific Collaboration. 2010, CQGra, 27, 084006

Hellier, C. 2001, Cataclysmic Variable Stars (Berlin: Springer)

Hopkins, P. F., Richards, G. T., \& Hernquist, L. 2007, ApJ, 654, 731

Howard, A. W., Marcy, G. W., Bryson, S. T., et al. 2012, ApJS, 201, 15

Iben, I., Jr., \& Tutukov, A. V. 1984, ApJS, 54, 335

Jakobsson, P., Hjorth, J., Malesani, D., et al. 2012, ApJ, 752, 62

Johnson, J. A., Clanton, C., Howard, A. W., et al. 2011, ApJS, 197, 26

Kasen, D. 2010, ApJ, 708, 1025
Katz, B., \& Dong, S. 2012, arXiv:1211.4584

Kelly, B. C., Bechtold, J., \& Siemiginowska, A. 2009, ApJ, 698, 895

Kopparapu, R. K., Hanna, C., Kalogera, V., et al. 2008, ApJ, 675, 1459

Kulkarni, S. R. 2005, arXiv:astro-ph/0510256

Kuroda, K., Ohashi, M., Miyoki, S., et al. 2003, CQGra, 20, 871

Kushnir, D., Katz, B., Dong, S., Livne, E., \& Fernández, R. 2013, ApJL, 778, L37

Lacy, J. H., Townes, C. H., \& Hollenbach, D. J. 1982, ApJ, 262, 120

Lawrence, A., Warren, S. J., Almaini, O., et al. 2007, MNRAS, 379, 1599

LIGO Scientific Collaboration, Virgo Collaboration, Abadie, J., et al. 2012, A\&A, 539, A124

Lodato, G., \& Rossi, E. M. 2011, MNRAS, 410, 359

MacLeod, C. L., Ivezić, Ž., Sesar, B., et al. 2012, ApJ, 753, 106

Maehara, H., Shibayama, T., Notsu, S., et al. 2012, Natur, 485, 478

Magorrian, J., \& Tremaine, S. 1999, MNRAS, 309, 447

Marconi, A., Risaliti, G., Gilli, R., et al. 2004, MNRAS, 351, 169

Martin, D. C., Fanson, J., Schiminovich, D., et al. 2005, ApJL, 619, L1

Matzner, C. D., \& McKee, C. F. 1999, ApJ, 510, 379

Maund, J., Reilly, E., \& Mattila, S. 2014, MNRAS, 438, 938

Metzger, B. D., \& Berger, E. 2012, ApJ, 746, 48

Montaruli, T. 2012, JPhCS, 375, 052032

Mushotzky, R. F., Edelson, R., Baumgartner, W., \& Gandhi, P. 2011, ApJL, 743, L12

Nakar, E., \& Piran, T. 2003, NewA, 8, 141

Nakar, E., \& Piran, T. 2011, Natur, 478, 82

Nakar, E., \& Sari, R. 2010, ApJ, 725, 904

Neill, J. D., Sullivan, M., Gal-Yam, A., et al. 2011, ApJ, 727, 15

Nikzad, S., Hoenk, M. E., Greer, F., et al. 2011, ApOpt, 51, 365

Nissanke, S., Sievers, J., Dalal, N., \& Holz, D. 2011, ApJ, 739, 99

Ofek, E. O., Fox, D., Cenko, S. B., et al. 2013c, ApJ, 763, 42

Ofek, E. O., Laher, R., Law, N., et al. 2012, PASP, 124, 62

Ofek, E. O., Lin, L., Kouveliotou, C., et al. 2013a, ApJ, 768, 47

Ofek, E. O., Rabinak, I., Neill, J. D., et al. 2010, ApJ, 724, 1396

Ofek, E. O., Sullivan, M., Cenko, S. B., et al. 2013b, Natur, 494, 65

Ofek, E. O., Zoglauer, A., Boggs, S. E., et al. 2014, ApJ, 781, 42

Pickles, A. J. 1998, PASP, 110, 863

Piran, T. 2004, RvMP, 76, 1143

Piro, A. L., Chang, P., \& Weinberg, N. N. 2010, ApJ, 708, 598

Piro, A. L., \& Nakar, E. 2012, arXiv:1211.6438

Polishook, D., Ofek, E. O., Waszczak, A., et al. 2012, MNRAS, 421, 2094

Poon, M. Y., \& Merritt, D. 2004, ApJ, 606, 774

Quimby, R. M., Aldering, G., Wheeler, J. C., et al. 2007, ApJL, 668, L99

Quimby, R. M., Kulkarni, S. R., Kasliwal, M. M., et al. 2011, Natur, 474, 487

Quimby, R. M., Yuan, F., Akerlof, C., \& Wheeler, J. C. 2013, MNRAS, 431, 912

Rabinak, I., Livne, E., \& Waxman, E. 2012, ApJ, 757, 35

Rabinak, I., \& Waxman, E. 2011, ApJ, 728, 63

Racusin, J. L., Karpov, S. V., Sokolowski, M., et al. 2008, Natur, 455, 183

Razzaque, S., Mészáros, P., \& Waxman, E. 2004, PhRvL, 93, 181101

Rhoads, J. E. 1997, ApJL, 487, L1

Robin, A. C., Reylé, C., Derrière, S., \& Picaud, S. 2003, A\&A, 409, 523

Rogerson, J. B., Spitzer, L., Drake, J. F., et al. 1973, ApJL, 181, L97

Sapir, N., Katz, B., \& Waxman, E. 2013, ApJ, 774, 79

Sari, R., Piran, T., \& Narayan, R. 1998, ApJL, 497, L17

Saxton, R. D., Read, A. M., Esquej, P., et al. 2012, A\&A, 541, A106

Schaefer, B. E. 2012, Natur, 485, 456

Schawinski, K., Justham, S., Wolf, C., et al. 2008, Sci, 321, 223

Schmidt, K. B., Rix, H.-W., Shields, J. C., et al. 2012, ApJ, 744, 147

Schneider, D. P., Richards, G. T., Hall, P. B., et al. 2010, AJ, 139, 2360

Sesar, B., Cohen, J. G., Levitan, D., et al. 2012, ApJ, 755, 134

Sesar, B., Svilković, D., Ivezić, Ž., et al. 2006, AJ, 131, 2801

Sesar, B., Vivas, A. K., Duffau, S., \& Ivezić, Ž. 2010, ApJ, 717, 133

Sholl, M. J., Ackerman, M. R., Bebek, C., et al. 2012, Proc. SPIE, 8446

Simon, T., Ayres, T. R., Redfield, S., \& Linsky, J. L. 2002, ApJ, 579, 800

Skrutskie, M. F., Cutri, R. M., Stiening, R., et al. 2006, AJ, 131, 1163

Smartt, S. J., Eldridge, J. J., Crockett, R. M., \& Maund, J. R. 2009, MNRAS, 395,1409

Soderberg, A. M., Berger, E., Page, K. L., et al. 2008, Natur, 453, 469

Stern, S. A., Parker, J. W., Feldman, P. D., et al. 2011, AJ, 141, 199

Strubbe, L. E., \& Quataert, E. 2009, MNRAS, 400, 2070

Svirski, G., Nakar, E., \& Sari, R. 2012, ApJ, 759, 108

Vanden Berk, D. E., Wilhite, B. C., Kron, R. G., et al. 2004, ApJ, 601, 692

van Eyken, J. C., Ciardi, D. R., Rebull, L. M., et al. 2011, AJ, 142, 60

van Eyken, J. C., Ciardi, D. R., von Braun, K., et al. 2012, ApJ, 755, 42

van Velzen, S., Farrar, G. R., Gezari, S., et al. 2011, ApJ, 741, 73

Voges, W., Aschenbach, B., Boller, T., et al. 1999, A\&A, 349, 389 
Wang, J., \& Merritt, D. 2004, ApJ, 600, 149

Waxman, E. 2011, arXiv:1101.1155

Waxman, E., \& Bahcall, J. 1997, PhRvL, 78, 2292

Welsh, B. Y., Wheatley, J. M., Heafield, K., et al. 2005, AJ, 130, 825

Welsh, B. Y., Wheatley, J. M., \& Neil, J. D. 2011, A\&A, 527, A15
Wheatley, J., Welsh, B. Y., \& Browne, S. E. 2012, PASP, 124, 552

Wilhite, B. C., Brunner, R. J., Grier, C. J., Schneider, D. P., \& vanden Berk, D. E. 2008, MNRAS, 383, 1232

Wright, E. L., Eisenhardt, P. R. M., Mainzer, A. K., et al. 2010, AJ, 140, 1868 York, D. G., Adelman, J., Anderson, J. E., Jr., et al. 2000, AJ, 120, 1579 